\title{
Hyperbaric oxygen alleviates the activation of NLRP-3-inflammasomes in traumatic brain injury
}

\author{
HUIHUI QIAN ${ }^{1 *}$, QINGHE $\mathrm{LI}^{2^{*}}$ and WODA SHI ${ }^{3}$ \\ Departments of ${ }^{1}$ Geriatrics, ${ }^{2}$ Nursing and ${ }^{3}$ Cardiothoracic Surgery, The Affiliated Yancheng Hospital, \\ Southeast University Medical College, Yancheng, Jiangsu 224001, P.R. China
}

Received April 1, 2016; Accepted March 7, 2017

DOI: $10.3892 / \mathrm{mmr} .2017 .7079$

\begin{abstract}
Growing evidence has demonstrated that the nucleotide-binding oligomerization domain-like receptor family pyrin domain containing 3 (NLRP-3) inflammasome-mediated inflammatory pathways have been involved in the secondary injury of traumatic brain injury (TBI). In the present study, the authors investigated the effects of hyperbaric oxygen (HBO) therapy on the NLRP-3 inflammasome pathway following TBI. Following the evaluation of motor deficits and brain edema, the therapeutic effects of $\mathrm{HBO}$ on interleukin (IL)- $1 \beta$ and IL-18 expression were assessed, as well as NLRP-3 inflammasome activation following TBI. HBO may improve motor score and reduce brain edema, accompanied with the reduction of IL-1 $\beta$ and IL-18 during the 7-day observation period. Furthermore, HBO suppressed mRNA and protein expression of NLRP-3-inflammasome components, especially reducing NLRP-3 expression in microglia. Thus, these results suggested that $\mathrm{HBO}$ alleviates the inflammatory response in experimental TBI via modulating microglial NLRP-3-inflammasome signaling.
\end{abstract}

\section{Introduction}

Traumatic brain injury (TBI), a major cause for trauma-related death and disability, may be divided into primary injury (a direct physical impairment of the brain) and secondary injury (a progressive brain damage mainly occurred within the first 12-24 h following injury) $(1,2)$. The mechanism of secondary injury after TBI has not been fully elucidated, but emerging studies support that the inflammatory response serves a curial role in the acute pathologic processes and longer-term neuronal

Correspondence to: Dr Woda Shi, Department of Cardiothoracic Surgery, The Affiliated Yancheng Hospital, Southeast University Medical College, 2 West XIndu Road, Yancheng, Jiangsu 224001, P.R. China

E-mail: yc3hpucheetah@aliyun.com

*Contributed equally

Key words: traumatic brain injury hyperbaric oxygen therapy, NLRP-3 inflammasome, microglia damage following TBI $(2,3)$. Thus, anti-inflammation therapy has been considered as an important therapeutic strategy for the treatment of TBI (4).

Growing evidence indicates that the inflammasome mediates the acute inflammatory response in a variety of brain injury including trauma (5-7). An inflammasome is an inducible high-molecular-weight complex that initiates inflammatory caspases and activates cytokines of the interleukin (IL)-1 family, including IL-1 $\beta$ and IL-18 $(8,9)$. The levels of the inflammasome proteins NACHT leucine-rich-repeat protein-1 (NALP1), apoptosis-associated speck-like protein containing caspase recruitment domain (ASC) and caspase-1 were significantly higher in the cerebrospinal fluid (CSF) of patients with severe or moderate cranial trauma, when compared with non-trauma controls (6). Furthermore, the CSF from TBI patients can activate the absent in melanoma 2 inflammasome and caspase-1, inducing pyroptosis in neighboring neurons (10). In addition, increased levels of the nucleotide-binding oligomerization domain-like receptor family pyrin domain containing 3 (NLRP3) gene coding for the NALP1 protein, ASC gene and caspase-1 protein were observed following TBI in a rat model (5). The traumatic contusion volume in mice was reduced during the treatment with an anti-ASC antibody immediately following TBI (7). These findings indicated that the inflammasome may be a potential target for treatment of TBI.

Hyperbaric oxygen (HBO) therapy, a well-established treatment in which a patient is administered at 1.5-3 absolute atmospheres (ATA) for 1-2 $\mathrm{h}$, is considered safe, and can provide obvious neuroprotection both in experimental and clinical studies, especially performed within 6 h post-TBI (11-13). $\mathrm{HBO}$ can promote neuronal metabolism, inhibit brain edema, protect the integrity of the blood-brain barrier (BBB), decrease cell apoptosis and inhibit the accumulation of inflammatory cells (14). However, its underlying mechanism is poorly understood.

Since $\mathrm{HBO}$ can attenuate IL-1 $\beta$-induced pro-inflammatory responses (15), the authors speculate about a potential role of $\mathrm{HBO}$ in regulation of inflammasome activation in TBI. To date, the most fully characterized inflammasome is the NLRP-3 inflammasome, which is involved in the neuroinflammatory response to TBI (5). In the present study, it is hypothesized that HBO has a neuroprotective effect on TBI via modulating NLRP-3 inflammasome activation. To verify this hypothesis, 
the authors compared the development of brain edema and motor deficits following TBI in mice with or without $\mathrm{HBO}$ therapy, and then assessed the therapeutic effects of $\mathrm{HBO}$ on IL-1 $\beta$ expression and NLRP-3 inflammasome activation. Furthermore, the cell type-specific expression of NLRP-3 inflammasome in TBI is also confirmed.

\section{Materials and methods}

Animals and treatment. A total of 280 male C57BL/6 mice (age, 10-12 weeks; weight, 22 \pm 5 g) were purchased from Shanghai SLAC Laboratory Animal Co., Ltd. (Shanghai, China) and housed in groups of five per cage under a temperature of $23 \pm 2^{\circ} \mathrm{C}$ and humidity of $55 \pm 5 \%$. They were maintained on a $12 \mathrm{~h}$ light/dark cycle with free access to food and water. The experimental protocols were approved by the Ethics Committee of Southern Medical University (Yancheng, China). The mice were familiarized to the housing conditions for a week prior to surgery.

The mice were randomly divided into six groups: Normal group (without any operation, $n=32$ ), sham group (with sham operation, $n=64)$, and four TBI groups at $6 \mathrm{~h}(\mathrm{n}=56), 1$ day $(n=32), 3$ days $(n=32)$, and 7 days $(n=64)$ post-operation. Every group further was randomly divided into two equal groups: Without $\mathrm{HBO}$ and with $\mathrm{HBO}$. According to preliminary analysis, there was no significant difference among sham groups at different time points (data not shown). Thus, the authors collected samples from mice belonging to the sham group 7 days following the sham operation.

Controlled cortical impact. The controlled cortical impact (CCI) of TBI was used. Briefly, mice were anesthetized with isoflurane and oxygen provided via a nose-cone mask, and then placed in a stereotactic frame. A $3 \mathrm{~mm}$-diameter craniotomy was performed over the left parietal cortex adjacent to the central suture between bregma and lambda. Injury was delivered using a $2.5 \mathrm{~mm}$-diameter tip with a velocity of $5.0 \mathrm{~m} / \mathrm{s}$ and a depth of $1.2 \mathrm{~mm}$. The temperature of mice was maintained at $37 \pm 0.5^{\circ} \mathrm{C}$ during the experiment. Sham-operated mice were anesthetized and only the left parietal craniotomy was made.

HBO treatment. HBO treatment was given for $1 \mathrm{~h}$ at 2.0 ATA in an experimental model hyperbaric chamber of transparent acrylic plastic (701 Space Research Institute, Beijing, China). The chamber was pressurized with $100 \%$ oxygen at a rate of $21 / \mathrm{min}$.

The mice without $\mathrm{HBO}$ were placed in the same chamber breathing room air. To minimize the effects of biological rhythm differences, all treatments were initiated at 2:00 pm. For repeated treatments, HBO was conducted once daily for 7 consecutive days.

Beam-balancing test. The beam-balancing test was used for neurological deficit scoring, as previously reported (16). Briefly, mice (16 mice from the normal group and the TBI at $6 \mathrm{~h}, 1$ and 3 days groups; 32 mice from the sham group and the TBI at 7 days group) were placed on a narrow wooden beam (6 mm wide and $120 \mathrm{~mm}$ in length). The status of maintaining balance was measured up to $60 \mathrm{sec}$ : 0 , walking easily and turning around freely; 1 , maintaining a stable posture during
$60 \mathrm{sec}$; 2, hugging or hooking the beam with its limb before $60 \mathrm{sec}$; and 3 , falling off of the beam.

Assessment of cerebral edema. Cerebral edema $(n=8)$ was quantified using the wet-dry method. Briefly, tissue from the ipsilateral cortex centered upon the impact site was immediately weighed (wet weight), then dehydrated at $65^{\circ} \mathrm{C}$ for 2 days. The sample was reweighed as dry weight. The percentage of tissue water content was calculated according to the following equation: $\% \mathrm{H}_{2} \mathrm{O}=[$ (wet weight - dry weight)/wet weight] x $100 \%$.

ELISA analysis of $I L-1 \beta$ and $I L-18$. The levels of IL-1 $\beta$ (cat. no. MLB00C) and IL-18 (cat. no. 7625) in tissues surrounding the cortical contusion site $(n=8)$ were performed by the specific ELISA kits according to the manufacturer's instructions (R\&D Systems, Inc., Minneapolis, MN, USA).

Reverse transcription-quantitative polymerase chain reaction. Total RNA was extracted from the ipsilateral cortex $(n=6)$ using TRIzol ${ }^{\circledR}$ reagent according to the manufacturer's protocol (Thermo Fisher Scientific, Inc., Waltham. MA, USA). Up to $1 \mu \mathrm{g}$ mRNA was reversed transcribed using the PrimeScript RT Master Mix kit (Takara Bio, Inc., Otsu, Japan) with a reaction volume of $20 \mu \mathrm{l}$ for $60 \mathrm{~min}$ at $42^{\circ} \mathrm{C}$. The reaction was terminated by heating at $75^{\circ} \mathrm{C}$ for $5 \mathrm{~min}$. qPCR was performed on cDNA using the ABI 9600 Detection system (Applied Biosystems; Thermo Fisher Scientific, Inc.), with a reaction volume of $10 \mu \mathrm{l}$. The sequences of the primers used were as follows: IL-1 $\beta$ (NM_008361.3) sense, 5'-TCATTG TGGCTGTGGAGAAG-3' and antisense, 5'-AGGCCACAG GTATTTTGTCG-3'; IL-18 (NM_008360.1) sense, 5'-ACT GTACAACCGCAGTAATAC-3' and antisense, 5'-AGTGAA CATTACAGATTTATCCC-3'; NLRP-3 (NM_145827.3) sense, 5'-CCCTTGGAGACACAGGACTC-3' and antisense, 5'-GAG GCTGCAGTTGTCTAATTCC-3'; ASC (NM_023258.4) sense, 5'-AGAGTACAGCCAGAACAGGA-3' and antisense, 5'-GATGGAACA AAGCTGAAGAG-3'; caspase-1 (NM_009807.2) sense, 5'-TATCCAGGAGGGAATATGTG-3' and antisense, 5'-ACAACACCACTCCTTGTTTC-3'; GAPDH (NM_008084.2) sense, 5'-AACGACCCCTTCATTGAC-3' and antisense, 5'-TCCACGACATACTCAGCAC-3'. Amplification conditions were as follows: Initially at $95^{\circ} \mathrm{C}$ for $5 \mathrm{~min}$, and then at $95^{\circ} \mathrm{C}$ for $5 \mathrm{sec}$ and at $58^{\circ} \mathrm{C}$ for $30 \mathrm{sec}$. This loop was repeated for a total of 38 cycles. The experiment was repeated three times, and the $2^{-\Delta \Delta \mathrm{Cq}}$ method was used for quantification of relative gene expression (17).

Western blot analysis. The homogenate from the pericontusional cortex $(n=6)$ was centrifuged $(12,000 \mathrm{x} \mathrm{g}, 10 \mathrm{~min}$, $4^{\circ} \mathrm{C}$ ) and the total supernatant protein was measured using a bicinchoninic acid assay kit (Pierce; Thermo Fisher Scientific, Inc.). The protein sample concentration was adjusted to $100 \mu \mathrm{g}$ and then separated by $10 \%$ SDS-PAGE before transferring the protein to nitrocellulose membranes which were blocked (TBS containing 10\% skim powder and $0.05 \%$ Tween-20) for $1 \mathrm{~h}$. The membranes were incubated with primary antibodies: anti-NLRP-3 (cat. no. AG-20B-0014-C100; 1:200; AdipoGen Corporation, San Diego, CA, USA), anti-ASC (cat. no. sc-514414; 1:500; Santa Cruz Biotechnology, Inc., Dallas, 
A

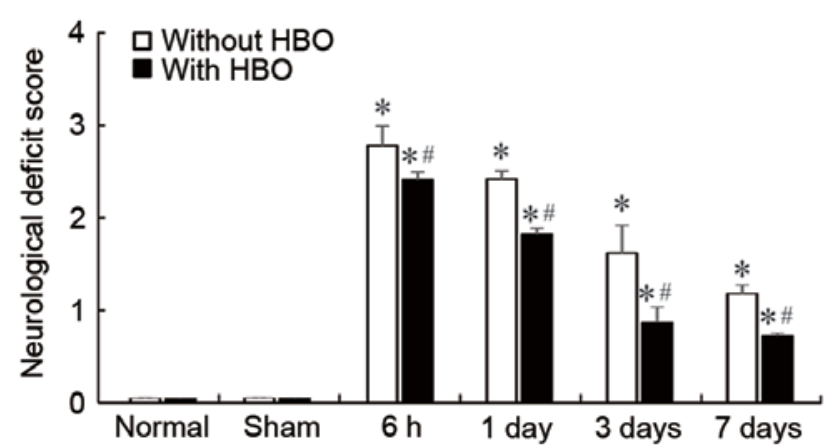

B

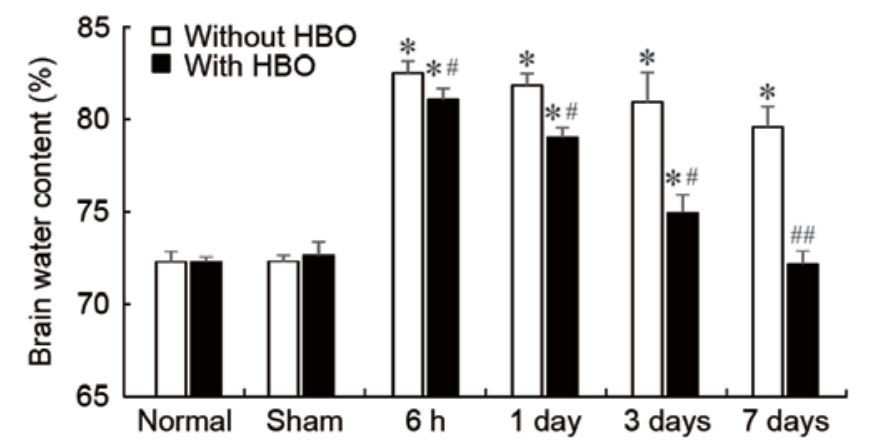

Figure 1. The scores of (A) beam balancing and (B) brain water content following traumatic brain injury in mice. Data are represented as mean \pm standard error of the mean. ${ }^{*} \mathrm{P}<0.05$ vs. corresponding normal group; ${ }^{\#} \mathrm{P}<0.05,{ }^{\# \#} \mathrm{P}<0.01$ vs. corresponding without $\mathrm{HBO}$ group. Beam balancing: $\mathrm{n}=16$ for the normal group and the TBI at 6 h, 1 and 3 days groups; $n=32$ for the sham group and the TBI at 7 days group; brain water content: $n=8$. TBI, traumatic brain injury; HBO, hyperbaric oxygen.

TX, USA), anti-caspase-1 (cat. no. AB1871; 1:1,000; Merck KGaA, Darmstadt, Germany), anti-IL-1 $\beta$ (cat. no. I3767; 1:1,000; Sigma-Aldrich; Merck KGaA) and anti- $\beta$-actin (cat. no. A5316; 1:1,000; Sigma-Aldrich; Merck KGaA) in TBS-T overnight at $4{ }^{\circ} \mathrm{C}$. Following incubation with the following horseradish peroxidase-conjugated secondary antibodies: Rabbit anti-mouse immunoglobulin (Ig) G H\&L (cat. no. ab6728; 1:1,000; Abcam, Cambridge, UK), goat anti-rabbit IgG H\&L (ab6721; 1:1,000; Abcam) and rabbit anti-goat IgG H\&L (ab6741; 1:1,000; Abcam) for $1.5 \mathrm{~h}$ at room temperature, protein bands were visualized using enhanced chemiluminescence western blotting detection reagents (Pierce; Thermo Fisher Scientific, Inc.) in a gel image analysis system (Tanon Science \& Technology Co., Ltd., Shanghai, China).

Immunofluorescence double labeling. Mice $(\mathrm{n}=4)$ were anaesthetized with pentobarbital sodium $(80 \mathrm{mg} / \mathrm{kg})$, and then perfused with $40 \mathrm{ml}$ saline and $40 \mathrm{ml} 4 \%$ paraformaldehyde. Following fixing in $4 \%$ paraformaldehyde at $4{ }^{\circ} \mathrm{C}$ overnight, the brains were maintained in $20 \%$ sucrose for 3 days, and in $30 \%$ sucrose for 3 days for dehydration. Coronal sections $(30 \mu \mathrm{m})$ of the pericontusional cortex were sliced using a freezing microtome (Leica CM1950; Leica Microsystems GmbH, Wetzlar, Germany). For double immunofluorescence, the sections were incubated overnight at $4^{\circ} \mathrm{C}$ with the following primary antibodies: Anti-NLRP-3 (1:200; cat. no. AG-20B-0014-C100; AdipoGen Corporation) and anti-neuronal nuclei (NeuN; cat. no. MAB377, 1:1,000; EMD Millipore, Billerica, MA, USA); anti-NLRP-3 and anti-glial fibrillary acidic protein (GFAP; cat. no. G6171, 1:1,000; Sigma-Aldrich; Merck KGaA); anti-NLRP-3 and anti-ionized calcium binding adaptor molecule 1 (Iba1; cat. no. 019-19741; 1:1,000; Wako Pure Chemical Industries, Ltd., Osaka, Japan). Sections were then incubated for $1.5 \mathrm{~h}$ at room temperature with the following fluorescent secondary antibodies: Alexa Fluor 594-conjugated goat anti-rabbit IgG (cat. no. R37117; 1:200; Invitrogen; Thermo Fisher Scientific, Inc.) and Alexa Fluor 488-conjugated goat anti-mouse IgG (cat. no. A-11001; 1:200; Invitrogen; Thermo Fisher Scientific, Inc.). Cell nuclei were labeled with 4',6-diamidino-2-phenylindole (DAPI; cat. no. D9542; 1:500; Sigma-Aldrich; Merck KGaA) for 5 min at room temperature. Stained sections were examined under a fluorescence microscope (Nikon Optical TE2000-S; Nikon Corporation, Tokyo, Japan).

Statistical analysis. Results were presented as mean \pm standard error of the mean and analyzed with one-way analysis of variance followed by post hoc Tukey's test using SPSS software (version 10.0; SPSS, Inc., Chicago, IL, USA). P<0.05 was considered to indicate a statistically significant difference.

\section{Results}

Treatment with HBO attenuated motor deficits. To evaluate neurological functional deficits, the beam-balancing test was conducted at different time points (6 h, 1 day, 3 days and 7 days) following TBI. As presented in Fig. 1A, normal (receiving no operation) and sham (receiving sham operation) mice demonstrated no significant movement deficits regardless of HBO treatment. TBI can cause a significant impairment in beam walk performance. The score of beam balancing was highest at $6 \mathrm{~h}$ post-TBI and then gradually deceased during 7 days following the operation, indicating that a spontaneous recovery from motor deficits occurred (Fig. 1A). Mice treated with HBO $1 \mathrm{~h}$ following TBI presented a significant amelioration of motor deficits compared to mice without $\mathrm{HBO}$ (Fig. 1A; $\mathrm{P}<0.05)$. Thus, $\mathrm{HBO}$ was effective for alleviating TBI-induced motor deficits.

Treatment with HBO alleviated cerebral edema. The development of posttraumatic cerebral edema following TBI exacerbates neurological deficits. As presented in Fig. 1B, TBI operation significantly increased the levels of brain water content during a 7-day observation period. Mice with HBO $1 \mathrm{~h}$ following TBI presented a significant reduction in water content, when compared to mice without $\mathrm{HBO}$ (Fig. 1B; $\mathrm{P}<0.05$ ). Thus, $\mathrm{HBO}$ can protect the brain parenchyma against cerebral edema post-TBI.

Treatment with $H B O$ decreased the levels of $I L-1 \beta$ and $I L-18$ in the pericontusional cortex. Time-course ELISA experiments (Fig. 2) demonstrated that the TBI operation induced the production of IL-1 $\beta$ and IL-18 significantly higher in sham groups over an observation period of $6 \mathrm{~h}-7$ days, when compared 
A

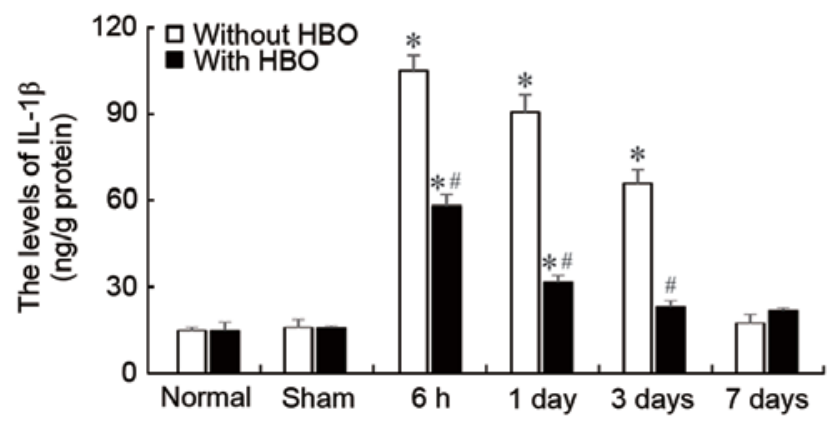

B

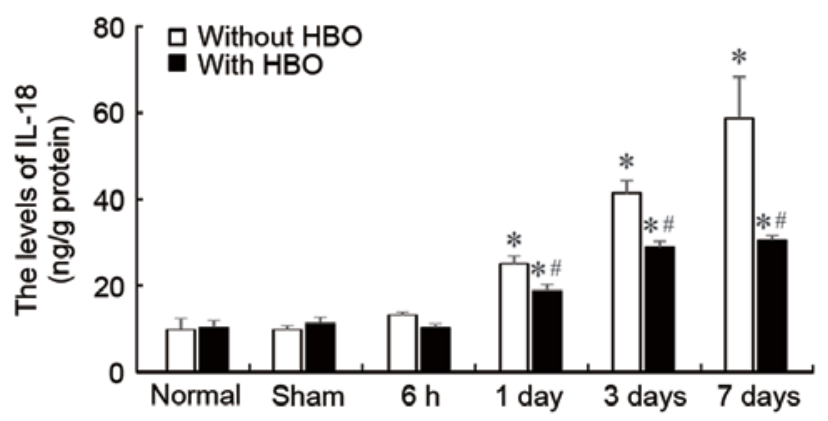

Figure 2. The levels of (A) IL-1 $\beta$ and (B) IL-18 in tissue surrounding the cortical contusion site. Data are represented as mean \pm standard error of the mean. ${ }^{*} \mathrm{P}<0.05$ vs. corresponding normal group; ${ }^{*} \mathrm{P}<0.05$ vs. corresponding without $\mathrm{HBO}$ group. $\mathrm{n}=8$. TBI, traumatic brain injury; HBO, hyperbaric oxygen; IL, interleukin.

A

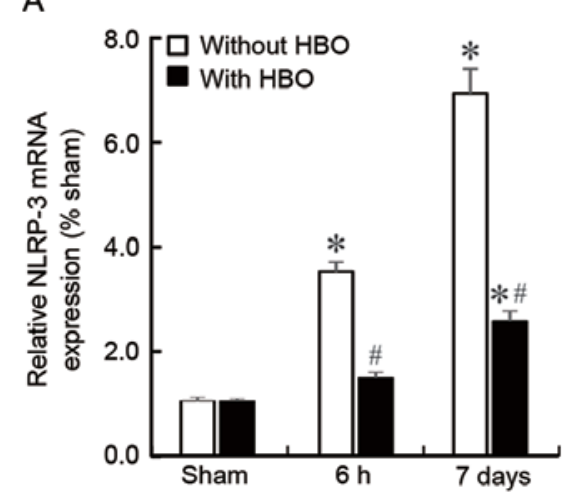

B

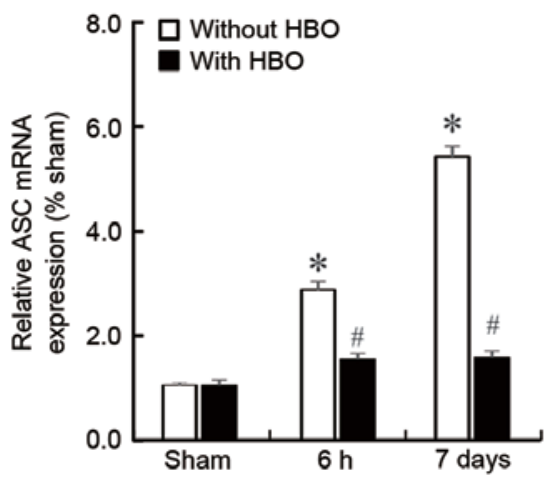

C

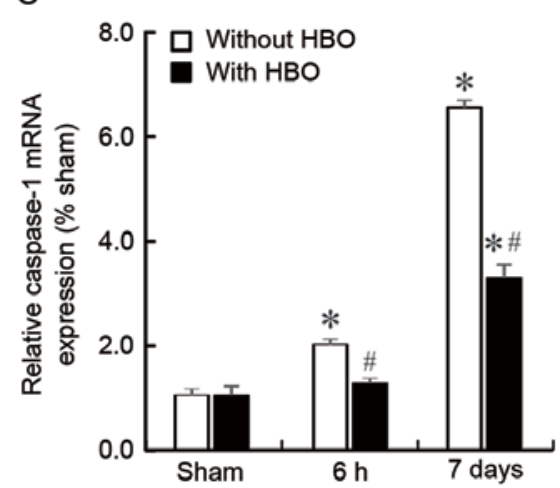

Figure 3. The mRNA expression of (A) NLRP-3, (B) ASC and (C) caspase-1 in tissue surrounding the cortical contusion site 6 h and 7 days post TBI. Data are represented as mean \pm standard error of the mean. ${ }^{*} \mathrm{P}<0.05$ vs. corresponding sham group; ${ }^{*} \mathrm{P}<0.05$ vs. corresponding without $\mathrm{HBO}$ group. $\mathrm{n}=6$. TBI, traumatic brain injury; HBO, hyperbaric oxygen; NLRP3, nucleotide-binding oligomerization domain-like receptor family pyrin domain containing 3; ASC, apoptosis-associated speck-like protein containing caspase recruitment domain.

without normal controls. The increase in cortical IL-1 $\beta$ levels rapidly reached a peak at $6 \mathrm{~h}$ and gradually decreased to the control level over a 7-day observation period (Fig. 2A), while the levels of IL-18 were gradually increased over the 7 days of operation (Fig. 2B). HBO treatment inhibited the production of these pro-inflammatory cytokines, both IL-1 $\beta$ and IL-18, during the observation period. As a result, two time points of $6 \mathrm{~h}$ and 7 days post-TBI were chosen for further experiments.

Treatment with $\mathrm{HBO}$ inhibited $m R N A$ expression of NLRP-3, $A S C$, and caspase- 1 . To investigate the effects of HBO treatment on the NLRP-3 inflammasome, the authors initially examined the mRNA levels of NLRP-3 inflammasome components, including NLRP-3, ASC and caspase-1. As indicated in Fig. 3, the mRNA expression of NLRP-3 (Fig. 3A), ASC (Fig. 3B) and caspase-1 (Fig. 3C) was significantly increased in the pericontusional cortex $6 \mathrm{~h}$ and 7 days following TBI operation. In addition, the levels of these NLRP-3 inflammasome components at $7 \mathrm{~d}$ post-TBI were elevated compared with those at $6 \mathrm{~h}$ post-TBI. HBO treatment inhibited the increase in NLRP-3 inflammasome mRNA expression levels in TBI-operated mice brain, irrespective of whether it was $6 \mathrm{~h}$ or 7 days post-operation (Fig. 3).

Treatment with $H B O$ suppressed protein expression of $N L R P-3, A S C$ and caspase-1. The authors further evaluated the protein expression of NLRP-3, ASC and cleaved caspase-1 in the pericontusional cortex $6 \mathrm{~h}$ and 7 days following TBI operation. The expression of NLRP-3 protein was weak in the sham group and increased significantly $6 \mathrm{~h}$ and 7 days following TBI operation $(\mathrm{P}<0.05$; Fig. 4A), which was suppressed by HBO treatment $(\mathrm{P}<0.05)$. Similar trends were also observed in the expression of ASC ( $\mathrm{P}<0.05$; Fig. 4B) and cleaved caspase-1 $(\mathrm{P}<0.05$; Fig. 4C).

Treatment with $\mathrm{HBO}$ reduced the immunofluorescence staining of NLRP-3 primarily in microglia. To clarify which cell type is primarily involved in the activation of the NLRP-3 inflammasome following TBI operation, the authors conducted double immunofluorescence labeling using NLRP-3/NeuN, NLRP-3/GFAP and NLRP-3/Iba1 in the 7 days group. As indicated in Fig. 5A, mice post-TBI had a loss of NeuN staining cells and an enhanced accumulation of NLRP-3 expression in the pericontusional cortex compared with the contralateral cortex. Furthermore, although both astrocytes and microglia were activated significantly, only microglia was accompanied with the strong expression of NLRP-3 in the pericontusional cortex. These results indicated that the activation of the NLRP-3 inflammasome was located in microglia in the cortex following TBI. Furthermore, HBO treatment significantly reduced the immunofluorescence staining of NLRP-3 in microglia (Fig. 5B). 

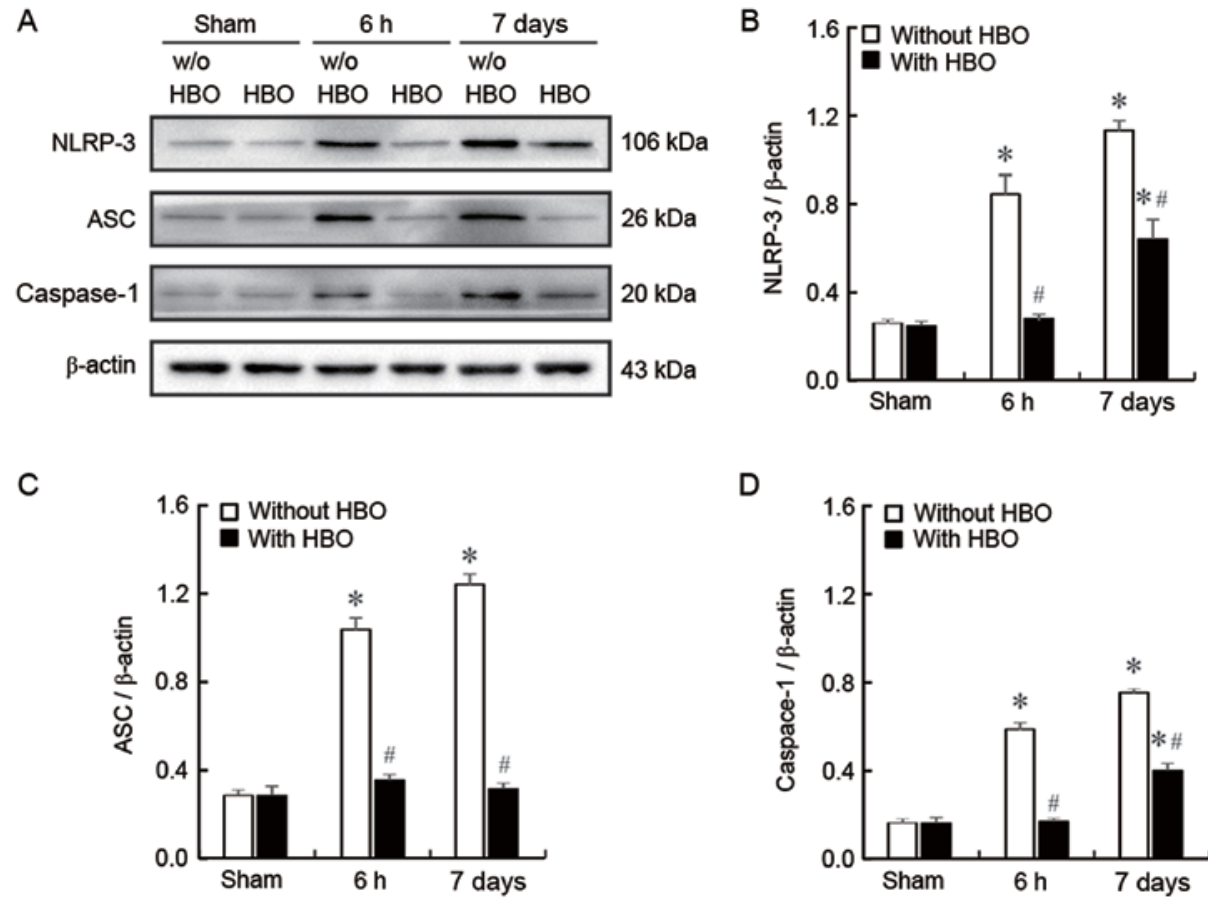

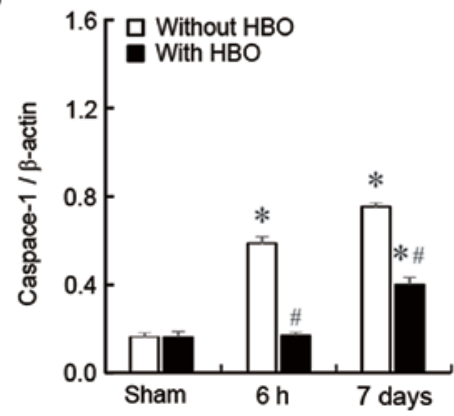

Figure 4. (A) Representative immunoblots of NLRP3, ASC and caspase-1. The protein expression of (B) NLRP-3, (C) ASC and (D) caspase-1 were measured in tissue surrounding the cortical contusion site $6 \mathrm{~h}$ and 7 days post TBI. Data are represented as mean \pm standard error of the mean. ${ }^{*} \mathrm{P}<0.05 \mathrm{vs}$. corresponding sham group; ${ }^{~} \mathrm{P}<0.05$ vs. corresponding without HBO group. $\mathrm{n}=6$. TBI, traumatic brain injury; HBO, hyperbaric oxygen; NLRP3, nucleotide-binding oligomerization domain-like receptor family pyrin domain containing 3; ASC, apoptosis-associated speck-like protein containing caspase recruitment domain.

A

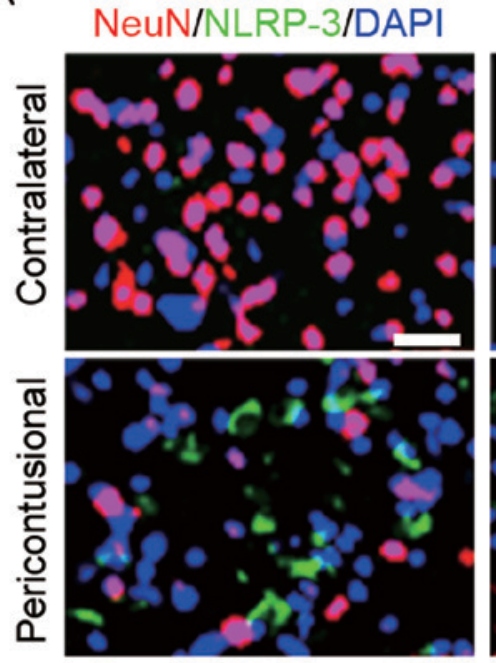

B

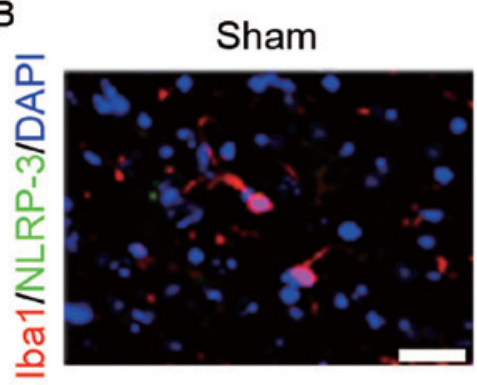

GFAP/NLRP-3/DAPI
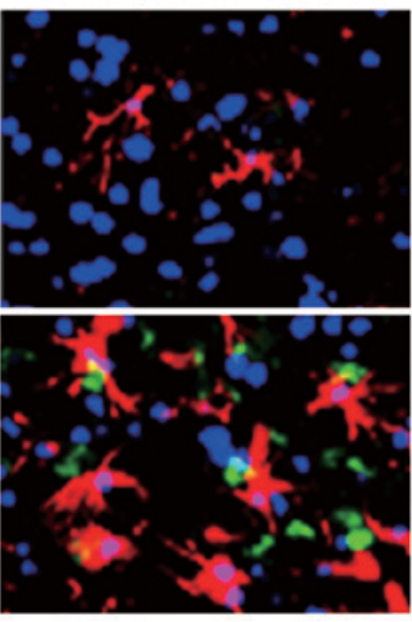

TBI

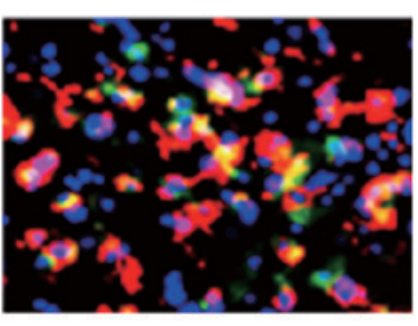

Iba1/NLRP-3/DAPI
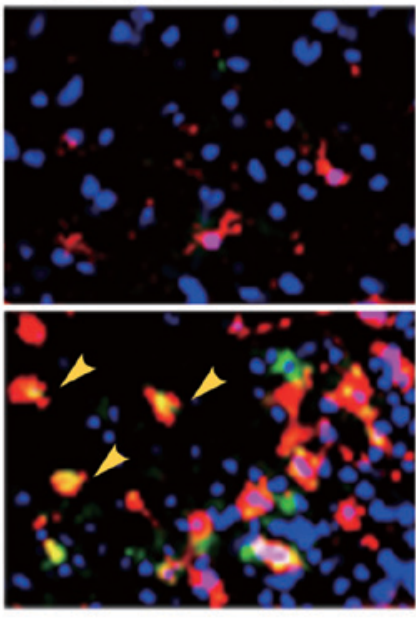

$\mathrm{TBI}+\mathrm{HBO}$

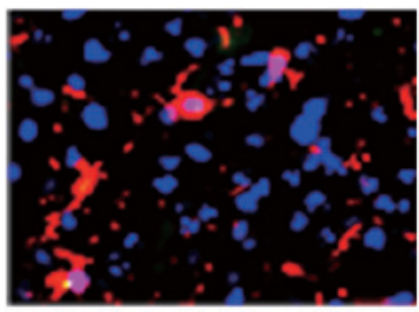

Figure 5. (A) Immunofluorescence double labeling for NLRP-3 (green) and cell special marker (red) in the contralateral (top) and pericontusional (bottom) cortex at 7 days following the operation. Left, NLRP-3 and NeuN; middle, NLRP-3 and GFAP; right, NLRP-3 and Iba-1. Yellow arrows indicate NLRP-3 positive microglial cells. Scale bars, $50 \mu \mathrm{m}$. (B) The effects of $\mathrm{HBO}$ on immunofluorescence double labeling for NLRP-3 (green) and Iba-1 (red) in the pericontusional cortex at 7 days post operation. Scale bars, $50 \mu \mathrm{m}$. Data are represented as mean \pm standard error of the mean. TBI, traumatic brain injury; HBO, hyperbaric oxygen; NLRP3, nucleotide-binding oligomerization domain-like receptor family pyrin domain containing 3; GFAP, glial fibrillary acidic protein; NeuN, neuronal nuclei; Iba-1, ionized calcium binding adaptor molecule 1. 


\section{Discussion}

The roles of hyperoxia during major brain injury remains controversial; however, previous studies in animal models and in humans have confirmed a theoretical basis for HBO therapy and attempted to explore its potential mechanism $(12,14)$. The present study demonstrated that in a mouse model of CCI, HBO treatment ameliorated the production of IL-1 $\beta$ and IL-18 via inhibiting NLRP-3 inflammasome activation.

Posttraumatic neuroinflammation is responsible for the development of secondary injury following TBI, and is associated with neurological deficits and edema development (3). As previously reported (9), it was also demonstrated that HBO improved motor function and reduced brain edema, accompanied with the reduction of IL-1 $\beta$ and IL-18. Interestingly, the increased expression of IL-1 $\beta$ in the pericontusional cortex reached a peak at $6 \mathrm{~h}$ and gradually decreased to the control level by day 7, while IL-18 were gradually increased over the 7 days of operation, as previously reported (5). IL-1 $\beta$ is an especially major pro-inflammatory cytokine in acute brain injury, and is capable of driving most of the inflammatory processes post-TBI (8). IL-1 $\beta$ can activate more microglia by further releasing more pro-inflammatory cytokines, and it also can increase the release of matrix metalloproteinase (MMP)-9 from astrocytes via activating the extracellular signal-regulated kinase pathway (18). MMPs mediate the breakdown of the blood brain barrier and neutrophil infiltration further promoting neuroinflammation (19). Thus, IL-1 $\beta$ is an aggravating factor in the early phase of TBI. On the other hand, growing evidences indicate that IL-18 serves a crucial role in mediating neuroinflammation and neurodegeneration under pathological conditions, such as hypoxic-ischemic brain injury and TBI (20). More specifically, the elevated levels of IL-18 is persistent following TBI, implicating its involvement in persistent long-term disability post-TBI (21). Considering the production of both IL-1 $\beta$ and IL-18 is derived by the inflammasome, the inflammasome has several different functions at different stages of TBI.

Activation of the NLRP-3 inflammasome has been implicated in TBI $(5,11)$. In the present study, it was observed that the NLRP-3-inflammasome components, such as NLRP-3, ASC and caspase-1, were increased at $6 \mathrm{~h}$ and 7 days post-TBI. Activation of the NLRP-3 inflammasome results in the inflammatory cascade, and then the secondary brain injury following TBI. In the CNS, neuroinflammation is mediated by microglia and astrocytes, which can release various pro-inflammatory cytokines including IL-1 $\beta$ (3). Activation of NLRP-3-inflammasomes has been reported in microglia and astrocytes $(22,23)$, while the NLRP1-inflammasomes, but not the NLRP-3-inflammasomes, are usually expressed by neurons $(24,25)$. By immunofluorescence, it was presented that NLRP-3 expression was primarily expressed in microglia, and not in astrocytes or neurons. Different from the research of Liu et al (5) in a rat model of TBI, the present study did not observe NLRP-3 positive neurons in the pericontusional cortex. As expected, $\mathrm{HBO}$ treatment prevented the increase in protein expression of NLRP-3, ASC and caspase-1 as well as IL-1 $\beta$ and IL-18 processing both $6 \mathrm{~h}$ and 7 days post-TBI, and significantly reduced the enhanced accumulation of NLRP-3 in microglia of pericontusional cortex. These finding confirmed the authors' hypothesis that $\mathrm{HBO}$ has a neuroprotective effect on TBI via modulating NLRP-3 inflammasome activation, especially in microglia.

HBO treatment directly increases the formation of reactive oxygen species (ROS), which may be a molecular mechanism underlying the effects of HBO on NLRP-3 inflammasome (26). However, the relationship between ROS and NLRP-3 inflammasome is still paradoxical. Some investigators have suggested that signaling by ROS drives NLRP-3 inflammasome formation (27), while others reported that an inducer of ROS can inhibit NLRP-3 inflammasome activation (28). Thus, additional research is required to elucidate the molecular mechanisms underlying the effects of $\mathrm{HBO}$ on the NLRP-3 inflammasome.

The authors' findings indicated that $\mathrm{HBO}$ alleviates the inflammatory response after TBI via inhibiting the activation of the NLRP-3-inflammasome signaling. However, the detailed mechanisms of HBO in regulation NLRP-3 inflammasome activation remain to be uncovered in future studies.

\section{References}

1. Kochanek PM, Jackson TC, Ferguson NM, Carlson SW, Simon DW, Brockman EC, Ji J, Bayir H, Poloyac SM, Wagner AK, et al: Emerging therapies in traumatic brain injury. Semin Neurol 35: 83-100, 2015.

2. Plesnila N: The immune system in traumatic brain injury. Curr Opin Pharmacol 26: 110-117, 2016.

3. Witcher KG, Eiferman DS and Godbout JP: Priming the inflammatory pump of the CNS after traumatic brain injury. Trends Neurosci 38: 609-620, 2015.

4. Corps KN, Roth TL and McGavern DB: Inflammation and neuroprotection in traumatic brain injury. JAMA Neurol 72: 355-362, 2015.

5. Liu HD, Li W, Chen ZR, Hu YC, Zhang DD, Shen W, Zhou ML, Zhu L and Hang CH: Expression of the NLRP3 inflammasome in cerebral cortex after traumatic brain injury in a rat model. Neurochem Res 38: 2072-2083, 2013.

6. Adamczak S, Dale G, de Rivero Vaccari JP, Bullock MR, Dietrich WD and Keane RW: Inflammasome proteins in cerebrospinal fluid of brain-injured patients as biomarkers of functional outcome: Clinical article. J Neurosurg 117: 1119-1125, 2012.

7. de Rivero Vaccari JP, Lotocki G, Alonso OF, Bramlett HM, Dietrich WD and Keane RW: Therapeutic neutralization of the NLRP1 inflammasome reduces the innate immune response and improves histopathology after traumatic brain injury. J Cereb Blood Flow Metab 29: 1251-1261, 2009.

8. Freeman LC and Ting JP: The pathogenic role of the inflammasome in neurodegenerative diseases. J Neurochem 136 (Suppl 1): S29-S38, 2016.

9. de Rivero Vaccari JP, Dietrich WD and Keane RW: Therapeutics targeting the inflammasome after central nervous system injury. Transl Res 167: 35-45, 2016.

10. Adamczak SE, de Rivero Vaccari JP, Dale G, Brand FJ III, Nonner D, Bullock MR, Dahl GP, Dietrich WD and Keane RW: Pyroptotic neuronal cell death mediated by the AIM2 inflammasome. J Cereb Blood Flow Metab 34: 621-629, 2014.

11. Wang GH, Zhang XG, Jiang ZL, Li X, Peng LL, Li YC and Wang Y: Neuroprotective effects of hyperbaric oxygen treatment on traumatic brain injury in the rat. J Neurotrauma 27: 1733-1743, 2010.

12. Camporesi EM and Bosco G: Mechanisms of action of hyperbaric oxygen therapy. Undersea Hyperb Med 41: 247-252, 2014.

13. Figueroa XA and Wright JK: Clinical results in brain injury trials using HBO2 therapy: Another perspective. Undersea Hyperb Med 42: 333-351, 2015.

14. Wang Y, Chen D and Chen G: Hyperbaric oxygen therapy applied research in traumatic brain injury: From mechanisms to clinical investigation. Med Gas Res 4: 18, 2014.

15. Chen X, Duan XS, Xu LJ, Zhao JJ, She ZF, Chen WW, Zheng ZJ and Jiang GD: Interleukin-10 mediates the neuroprotection of hyperbaric oxygen therapy against traumatic brain injury in mice. Neuroscience 266: 235-243, 2014. 
16. Dixon CE, Lyeth BG, Povlishock JT, Findling RL, Hamm RJ, Marmarou A, Young HF and Hayes RL: A fluid percussion model of experimental brain injury in the rat. J Neurosurg 67: 110-119, 1987.

17. Livak KJ and Schmittgen TD: Analysis of relative gene expression data using real-time quantitative PCR and the 2(-Delta Delta C(T)) method. Methods 25: 402-408, 2001.

18. Ralay Ranaivo H, Zunich SM, Choi N, Hodge JN and Wainwright MS: Mild stretch-induced injury increases susceptibility to interleukin-1 $\beta$-induced release of matrix metalloproteinase-9 from astrocytes. J Neurotrauma 28: 1757-1766, 2011.

19. Vafadari B, Salamian A and Kaczmarek L: MMP-9 in translation: From molecule to brain physiology, pathology, and therapy. J Neurochem 139 (Suppl 2): S91-S114, 2016.

20. Felderhoff-Mueser U, Schmidt OI, Oberholzer A, Bührer C and Stahel PF: IL-18: A key player in neuroinflammation and neurodegeneration? Trends Neurosci 28: 487-493, 2005.

21. Ciaramella A,Della Vedova C,Salani F,Viganotti M,D'Ippolito M, Caltagirone C, Formisano R, Sabatini U and Bossù P: Increased levels of serum IL-18 are associated with the long-term outcome of severe traumatic brain injury. Neuroimmunomodulation 21: 8-12, 2014.

22. Halle A, Hornung V, Petzold GC, Stewart CR, Monks BG, ReinheckelT, Fitzgerald KA, LatzE, Moore KJ and GolenbockDT: The NALP3 inflammasome is involved in the innate immune response to amyloid-beta. Nat Immunol 9: 857-865, 2008.
23. Jha S, Srivastava SY, Brickey WJ, Iocca H, Toews A, Morrison JP, Chen VS, Gris D, Matsushima GK and Ting JP: The inflammasome sensor, NLRP3, regulates CNS inflammation and demyelination via caspase-1 and interleukin-18. J Neurosci 30: 15811-15820, 2010.

24. Silverman WR, de Rivero Vaccari JP, Locovei S, Qiu F, Carlsson SK, Scemes E, Keane RW and Dahl G: The pannexin 1 channel activates the inflammasome in neurons and astrocytes. J Biol Chem 284: 18143-18151, 2009.

25. de Rivero Vaccari JP, Lotocki G, Marcillo AE, Dietrich WD and Keane RW: A molecular platform in neurons regulates inflammation after spinal cord injury. J Neurosci 28: 3404-3414, 2008.

26. Matsunami T, Sato Y, Hasegawa Y, Ariga S, Kashimura H, Sato $\mathrm{T}$ and Yukawa M: Enhancement of reactive oxygen species and induction of apoptosis in streptozotocin-induced diabetic rats under hyperbaric oxygen exposure. Int J Clin Exp Pathol 4: 255-266, 2011.

27. Tschopp J and Schroder K: NLRP3 inflammasome activation: The convergence of multiple signalling pathways on ROS production? Nat Rev Immunol 10: 210-215, 2010.

28. Maier NK, Crown D, Liu J, Leppla SH and Moayeri M: Arsenic trioxide and other arsenical compounds inhibit the NLRP1, NLRP3, and NAIP5/NLRC4 inflammasomes. J Immunol 192: 763-770, 2014. 